\title{
Comparison of the Hybrid Locking Plate, Standard Dynamic Compression Plate, and Standard Dynamic Compression Plate Augmented with Bone Cement for Fixation of Osteoporotic Humeral Shaft Fractures: A Cadaveric Biomechanical Study
}

\author{
Irewin A. Tabu, Jose Joefrey F. Arbatin, Jr. and Rafael C. Bundoc \\ Department of Orthopedics, College of Medicine and Philippine General Hospital, University of the Philippines Manila
}

\begin{abstract}
Background. Studies comparing the relative strength of polymethylmethacrylate (PMMA) augmented fixation, standard plating and locked compression plate (LCP) system are few. The use of either the bone cement-augmented dynamic compression plate or the Hybrid LCP constructs may provide an additional tool for the treatment of fractures in patients with osteoporosis.
\end{abstract}

Methods. Eighteen (18) osteoporotic cadaveric humeral bones were assigned randomly to each of three groups (Dynamic Compression Plate [DCP], DCP augmented with bone cement, and the Hybrid LCP system) and tested in anterior-posterior bending and torsion/external rotation. The load to failure values were obtained and the results for each specimen compared.

Results. Significant differences were observed between the standard DCP and Hybrid LCP group ( $p$-value $=0.012$ ), and in the cement-augmented and Hybrid LCP group ( $p$-value=0.099) in torsion/external rotation loading. No significant difference was observed between the standard DCP and bone-cement augmented group ( $p$-value $=0.248$ ). No significant difference was observed among the three groups in terms of stiffness ( $p$ value $=0.3868$ ) in the four-point anterior-posterior bending modality. Screw pull-out of the implant was observed only in the regular DCP group in torsion/external rotation loading stress.

Conclusion. Significant differences were seen between the three constructs in torsion/external rotation but not in anteriorposterior four-point bending. Bone failure, but not screw pullout, was seen in the Hybrid LCP and bone cement-augmented DCP groups in torsion. This study showed that the LCP system and the bone cement-augmented constructs may provide greater screw purchase to the osteoporotic humerus.

Key Words: humeral shaft fracture, osteoporosis, dynamic compression plate, polymethylmethacrylate (PMMA), locking compression plate $(L C P)$

\footnotetext{
Corresponding author: Irewin A. Tabu, MD

Department of Orthopedics

Philippine General Hospital

University of the Philippines Manila

Taft Avenue, Ermita, Manila 1000 Philippines

Telephone: +6325548466

Email: absmd94h@yahoo.com
}

Introduction

The management of patients with osteoporotic fractures requires meticulous preoperative planning and surgical technique due to the poor quality of bone. Bone failure, not implant breakage, is the primary mode of failure of internal fixation in osteoporotic bone. ${ }^{1}$ Because bone mineral density correlates with the holding power of screws, osteoporotic bone often lacks the strength to hold plates and screws securely. Achieving stable internal fixation for fractures in osteoporotic bone can be problematic but is central to effective care because it facilitates earlier rehabilitation and subsequent return of function. ${ }^{1}$

Osteoporosis is a systemic disease characterized by decreased bone mass and deteriorated bone micro architecture. In the elderly ( $\geq 65$ years), it is a contributing factor in $75 \%$ of fractures caused by low-energy falls. ${ }^{1}$

In the United States, 1.5 million fractures are reported annually, most from low-energy falls, including 300,000 proximal femur fractures, 250,000 distal radius fractures, and 300,000 fractures in other bones affected by osteoporosis. Fifty percent of women and $18 \%$ of men older than 50 years will sustain an osteoporotic fracture. Although US\$13.8 billion is spent annually to manage these fractures, $<50 \%$ of hip fracture patients recover fully after treatment. These statistics emphasize the need for skilled fracture care for osteoporotic patients. ${ }^{1}$

The advent of dynamic compression plates (Figure 1) introduced an effective treatment for humeral diaphyseal fractures in normal adults. Plate and screw constructs follow the tenets put forth by the A-O group in the late 1950s. The desired result of this intervention was anatomic bone union. Complications using these techniques included delayed union, nonunion, refractures after device removal, bone failure and infection. ${ }^{2}$ Consequently, an effort has been made to reduce the number of complications utilizing an improved understanding of the roles of gap strain and tissue vascularity. ${ }^{2}$ However, significant problems arise when the patient also suffers from osteopenia/osteoporosis. Instability of a construct due to inadequate screw purchase into the adjacent cortices can result in poor fracture stabilization, mechanical failure, and persistent nonunion. Complications 
such as screw pull-out result in $10 \%$ to $25 \%$ loss of fixation. ${ }^{1}$ To facilitate primary bone healing, one must ensure rigid fracture stabilization through firm bone contact and compression of the bone ends. Sufficient mechanical stability is essential to reduce strain at the fracture site and allow biologic repair. ${ }^{1}$

A major change in the rationale of fracture fixation occurred with development of locked bridging internal fixation in the late 1990s. When using this implant and the theory of "bridging plate osteosynthesis", fracture union occurred by secondary, not primary, bone healing as with rigid internal fixation. This initial locked plate design created the first fixed screw-plate single composite beam construct, much like a conventional external fixture. Further refinements to the PC-Fix concept led to the design and manufacture of the locked compression plate (LCP, Synthes) (Figure 2). The LCP provided angular and axial stability which decreased or eliminated the need for exact plate contouring, thereby minimizing the risk of primary loss of reduction. ${ }^{2}$ Initial biomechanical and clinical studies regarding LCP reported higher load to failure rates compared with the standard DCP. ${ }^{2-6}$ Gardner et al. compared osteoporotic fresh frozen human cadaver radius fixed with DCP and LCP. In torsion, LCP failed at $60 \%$ greater Newton-cycles than DCP (1473 vs. 918, p<0.05). ${ }^{7}$

Locked plates may increasingly be indicated for indirect fracture reduction, for diaphyseal/metaphyseal fractures in osteoporotic bone, bridging severely comminuted fractures, and for the plating of fractures where anatomical constraints prevent plating on the tension side of the bone. However, the use of the LCP has not been without controversy. ${ }^{2}$ Given the higher cost of the implant and lack of definitive long term studies especially in osteoporotic fractures, the use of this system is still being questioned by many orthopedic surgeons.

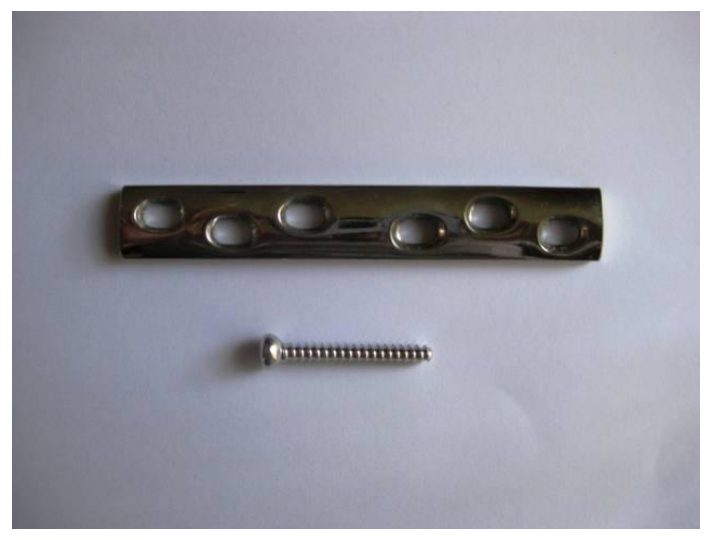

Figure 1. The standard 4.5 broad dynamic compression plate and its cortical screw

Other alternatives are still being utilized by orthopedic surgeons to augment fracture fixation in osteoporotic humeral shaft fractures in order to prevent complications such as implant failure and screw pull-out. One such alternative is the use of polymethylmethacrylate (PMMA). Although PMMA has relatively poor adhesion to bone, its intrusion into the cancellous structure results in a much stronger composite after the cement polymerizes. ${ }^{1,2}$ The use of PMMA has been suggested to augment fixation in hip and supracondylar femoral fractures ${ }^{8}$ and to increase the relative strength of fixation, but its use in the humerus has been limited. There are few studies comparing the relative strength of PMMA-augmented fixation in the osteoporotic humerus compared to the standard plating system and the LCP system. Also, most of these studies use synthetic or saw bone models in comparing the biomechanical characteristics of these implants. $3,5,8,9$

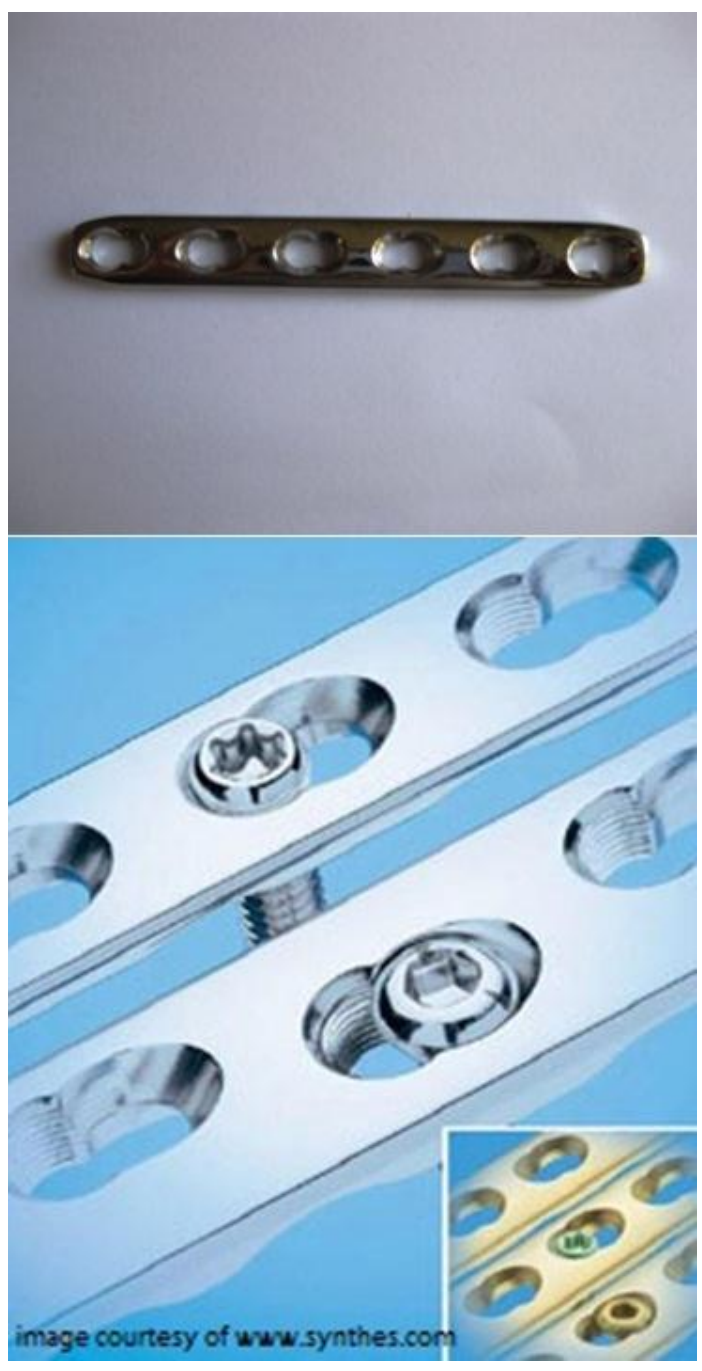

Figure 2. The limited contact locking compression plate and its screw

Elderly patients with osteoporotic fractures treated with inadequate fixation increases the risk of thromboembolic disease, pulmonary complications, and generalized musculoskeletal deterioration for which recovery is 
unlikely. ${ }^{1}$ Thus, it is vital that the surgical procedure be kept as simple as possible to minimize surgical time, blood loss, and physiologic stress. Although plate osteosynthesis is one of the most common methods of fixation for humeral shaft fractures, the choice of the type of plate for osteoporotic fractures is still made largely on the basis of anecdotal reports and clinical experience with mechanical failure. This study will provide objective biomechanical evidence which would allow surgeons to choose a construct with sufficient stability. ${ }^{10}$ Furthermore, considering the high cost of implants, obtaining objective biomechanical data regarding different fixation constructs in the study may provide orthopedic surgeons cost-effective alternatives in treating diaphyseal fractures of the humerus in osteoporotic patients.

\section{Objectives}

\section{A. General Objective}

The goal of this study is to compare the biomechanical properties of three constructs (standard DCP, bone cementaugmented DCP [Figure 3], and the Hybrid LCP system) in the fixation of osteoporotic fractures of the humerus.

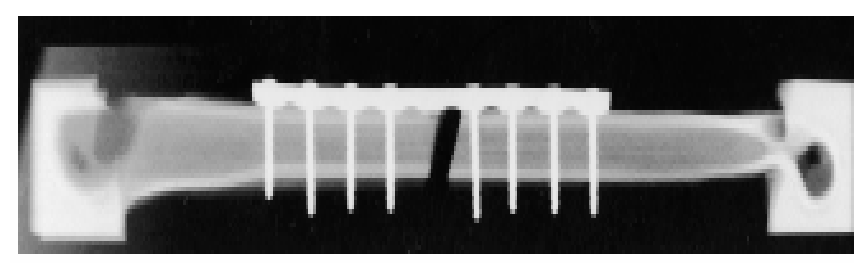

Figure 3. Standard 4.5 dynamic compression plate fixation augmented with bone cement

\section{B. Specific Objectives}

1. To compare the stiffness, in terms of load to failure, afforded by the three constructs when subjected to external rotation/torsion loading test.

2. To compare the stiffness, in terms of load to failure, afforded by the same three constructs when subjected to anterior-posterior four-point bending loading test.

3. To determine if screw pull-out of the implant would occur in these three constructs within the elastic range of loading and before bone failure occurs.

\section{Methods}

\section{A. Research Setting}

Materials were gathered from human cadavers from the UP College of Medicine Anatomy Laboratory. Biomechanical testing was performed at the Department of Orthopedics Biomechanical Laboratory (UP-PGH Spine Unit Center) and at the Orthopedic International Plant at Cabuyao, Laguna.

\section{B. Sample Size}

There is no local data comparing the biomechanical properties of the standard DCP, the bone cement-augmented DCP, and the Hybrid LCP system in the fixation of osteoporotic humeral shaft fractures; thus, this will be a pilot study. The biomechanical study by Rubel et al. ${ }^{11}$ comparing different plate constructs in humeral shaft fracture fixation utilized six humeral shafts each for the four groups compared. Eighteen cadaveric humeral shafts were utilized for our study; nine were tested for external rotation/torsion and nine for anterior-posterior four-point bending. Three bones were assigned randomly to each of the three groups. ${ }^{11}$

\section{Specimens}

Mechanical tests were performed on 18 cadaveric female adult humeral shafts which were divided between the two loading modalities (nine each for the two testing modes) and was then assigned to the three groups of implant construct $(n=3)$. The surrounding soft tissue and muscle was dissected off the humeral shafts, with care being taken to preserve the bone. The bone mineral density was measured by dual-energy X-ray absorptiometry to confirm that they will at least be moderately osteoporotic (values between 0.200 and 0.400$),{ }^{4,12}$ and the presence of pathologic lesions was ruled out by viewing plain radiographs (Figure 4). The plate was then applied to the lateral side of the humeral shaft. The center of the bone was determined, and a 5-mm transverse-gap osteotomy was performed at the midshaft level (Figure 5).

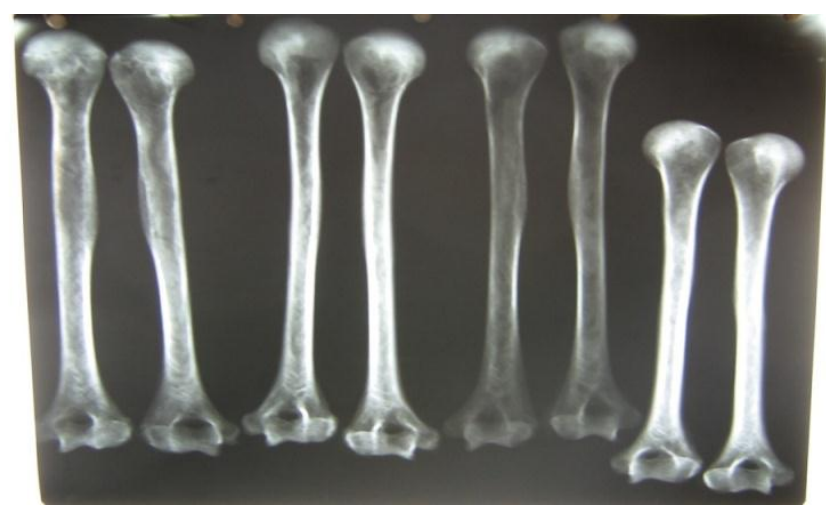

Figure 4. Sample radiographs of the specimen osteoporotic humerus

\section{Implants}

Three plate constructs were tested: Group I specimens were fixed with a six-hole $4.5-\mathrm{mm}$ broad dynamic compression plate, Group II with a six-hole 4.5-mm dynamic compression plate augmented with polymethylmetacrylate (PMMA) bone cement, and Group III with a six-hole 5.0-mm Hybrid Locking compression plate. 


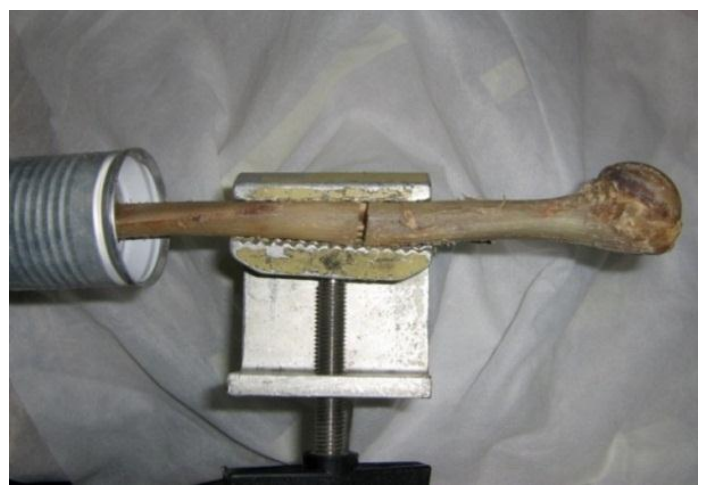

Figure 5. 5-mm transverse gap osteotomy made at the center of the humerus

\section{E. Method of Implantation}

Broad 4.5 regular stainless steel dynamic compression plates using $4.5-\mathrm{mm}$ cortical screws inserted into $3.5-\mathrm{mm}$ pilot holes were used in group I. Group II involved the same technique as group I but with bone cement augmentation performed on each of the pilot holes before screw insertion. Similarly, stainless steel locking compression plates using 5.0-mm cortical screws inserted into $4.0-\mathrm{mm}$ pilot holes were used for the LCP group, group III. The hybrid variation involved the use of the regular $4.5-\mathrm{mm}$ cortical screw in the two screw holes immediately proximal and distal to the fracture site, but 5.0-mm locking cortical screws were used in all the remaining screw holes. All screws were tightened to the same torque value, measured with a torque screw driver. Specimens were then tested in a materials testing machine (Enduratec Universal Testing Machine) in two loading modalities: 1) torsion/external rotation and 2) fourpoint anterior-posterior bending. The three groups of construct $(n=3)$ underwent testing in each of the two loading modalities. The specimens were then inspected to see if screw pull-out of the implant would occur before bone failure in torsion/external rotation loading. All laboratory fracture models were tested for biomechanical characteristics of the constructs in terms of load to implant failure.

\section{F. Bone Cement Augmentation Technique}

The PMMA powder and liquid were cooled to slow down polymerization. The components were then mixed and the liquid cement placed into a $10-\mathrm{ml}$ syringe with the tip widened by drilling it out with a $3.5-\mathrm{mm}$ drill. The cement was then injected into the stripped screw holes, after which the screws were inserted but incompletely tightened. The screws were fully tightened as soon as the cement had set. ${ }^{1}$

\section{G. Mechanical Testing}

The bone-implant constructs were loaded with the materials testing machine in the elastic range to determine their stiffness. Each specimen was tested in torsion (external rotation) and anterior-posterior bending. A four-point bending test was used for the anterior-posterior tests. The loader was coupled to a universal joint to allow for selfalignment during the test (Figure 6). Load-deflection curves were recorded for each of the three specimens in the three groups. A load was applied until implant failure was observed in each of the constructs. External rotation was achieved by placing the specimens in the load-frame vertically and pinning them into the grips. A custom designed jig was applied to each metaphyseal end which centered the bone in the torsion actuator of the testing machine (Figure 7). External rotation torque was applied to the composite at a rate of $26.5 \mathrm{in} / \mathrm{lb}$ to a maximum of 132 in/lb. All tests were carried out until implant failure was observed for all the constructs. The data was recorded on a computer with use of Lab tech data acquisition software. ${ }^{4,13}$

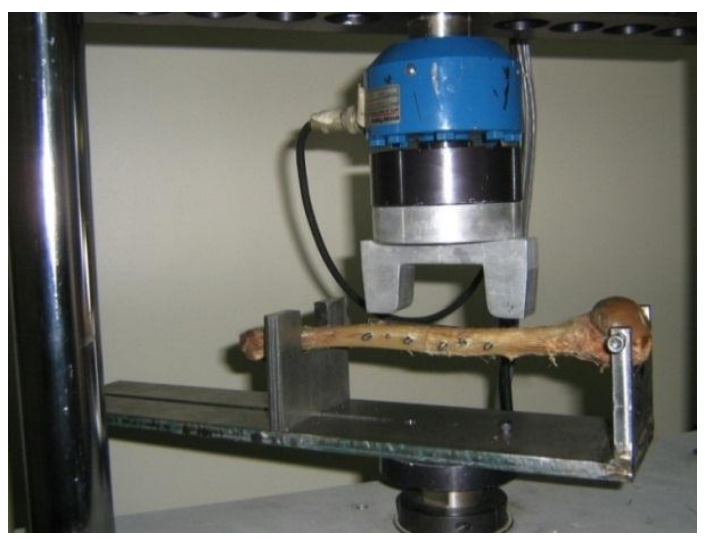

Figure 6. Set-up for anterior-posterior four-point bending test

\section{Ethical Considerations}

Only cadavers from the UP College of Medicine Anatomy Laboratory were utilized with the approval of the Chairman of the Department of Anatomy, ensuring that the cadavers were already abandoned by their respective families. All cadaveric humeral shafts were returned to the Department of Anatomy after the biomechanical testing for proper burial of the bones.

\section{Data Analysis}

Data retrieved from external rotation torque and fourpoint anterior-posterior bending tests were analyzed using a repeated-measures analysis of variance (ANOVA) with an alpha level of 0.05. Scheffe Multiple Comparisons tests were performed for the results which showed significant difference. Assumptions of the power analysis included: alpha $=0.05$. The sample size computation for the study was based on the formula by Montgomery DC, Design and Analysis of Experiments. Analysis = repeated measures analysis of variance was followed by Scheffe Multiple Comparisons tests (Stata, version 6; StataCorp, Texas). 


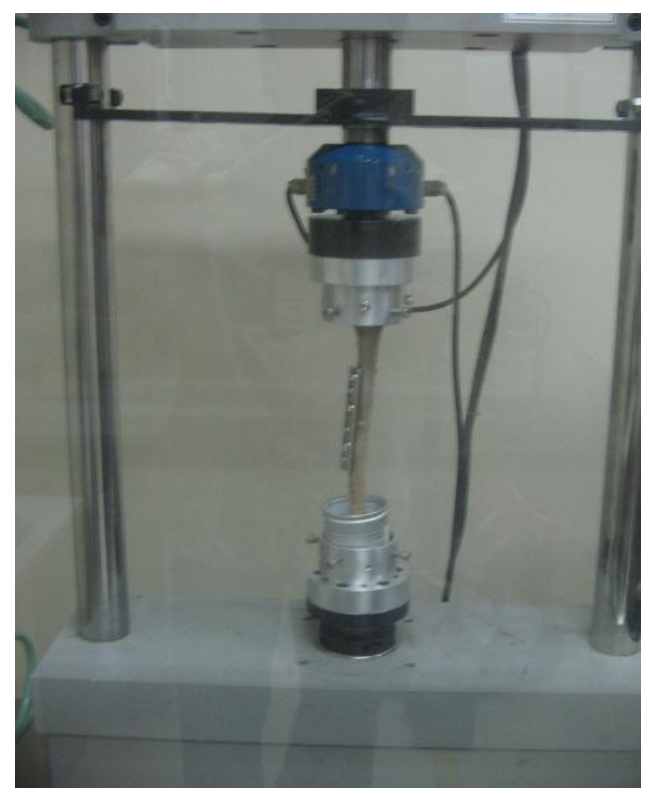

Figure 7. Set-up for the torsion/external rotation testing

\section{Results}

Data from the biomechanical testing was encoded and analyzed using the Repeated Measures Analysis of Variance (ANOVA) in order to determine the effect of the fixation method (standard DCP, DCP with PMMA bone cement augmentation and Hybrid LCP) on the stiffness of the construct in terms of load to failure in the two loading modalities. Significant results were further tested using Scheffe Multiple Comparisons Test.

In the torsion/external rotation loading modality, significant differences were observed among the three groups (p-value=0.(7) Staticticallv sionificant differences were observed specifically between the standard DCP group and the Hybrid LCP group ( $p$-value=0.012), as well as in the cement-augmented group and the Hybrid LCP group ( $\mathrm{p}-$ value $=0.099$ ). However, no significant statistical difference was observed between the standard DCP group and the bone cement augmented group ( $\mathrm{p}$-value $=0.248$ ) despite the fact that the bone cement augmented group had higher load to failure values as compared with the standard DCP group. Results of the biomechanical tests in torsion/external rotation loading of the three groups of implant constructs are summarized in a bar graph (Figure 8).

In the four-point anterior-posterior bending loading modality where all specimens were loaded until bone failure (Figure 9), no statistically significant difference was observed among the three groups in terms of stiffness ( $p$ value $=0.3868$ ). However, the Hybrid LCP group had higher load to failure values compared to both the standard DCP and cement-augmented group. The cement-augmented group showed higher load to failure values compared to the standard DCP group (Figure 10).

Screw pull-out of the implant was observed only in the regular DCP group and not in the cement-augmented and Hybrid LCP group in torsion/external rotation loading stress. Screw pull-out was seen to occur in the screws proximal to the fracture gap in two of the three bone specimens in the standard DCP group (Figure 11). Bone failure (actual breakage of the bone) was seen in all the specimens in both loading modalities. All failures were observed to originate from the bone component of the constructs. Longitudinal split fractures as well as spiral bone fractures were the patterns of bone failure seen in our testing (Figure 12). None of the implants were deformed after the testing and all were observed to be of the same shape and

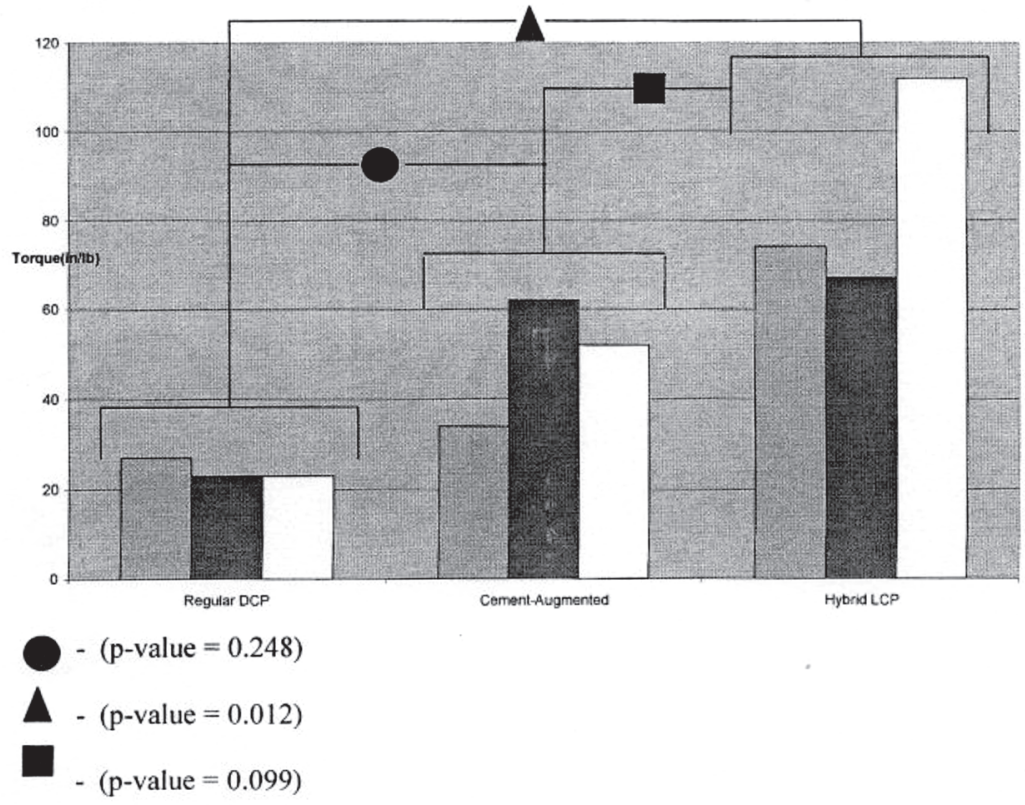

Figure 8. Mean load failure values in external rotation by implant construct 


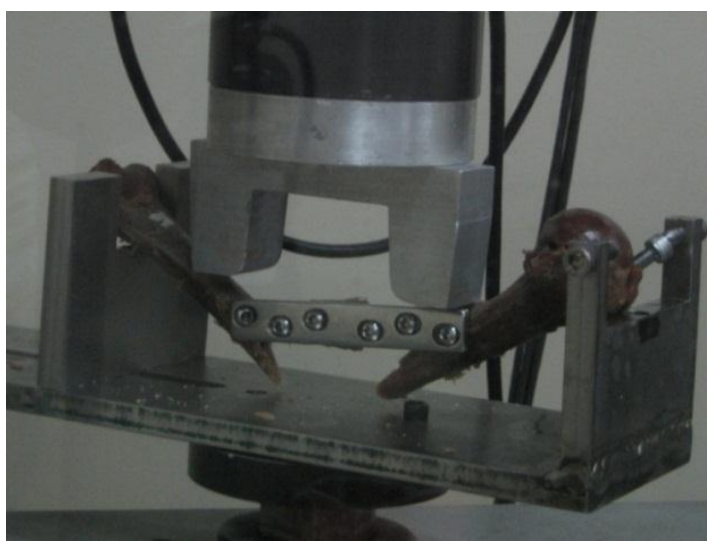

Figure 9. Pattern of bone failure in the anterior-posterior four-point bending test

\section{Discussion}

Significant problems arise when the patient with a fracture has established osteopenia/osteoporosis. As previously mentioned, an unstable construct resulting from inadequate screw purchase into adjacent cortices can result in poor fracture stabilization, mechanical failure, and persistent nonunion. Complications such as screw pull-out result in $10 \%$ to $25 \%$ loss of fixation. ${ }^{1}$ Previous studies show that locking plate constructs are superior to unlocked plates and screws when tested under torsion in an unstable osteoporotic diaphyseal fracture model. ${ }^{3}$ Also, locked hybrid constructs were shown to be mechanically similar to an alllocked screw construct. ${ }^{3}$ There are few studies comparing the relative strengths of the different constructs and most of these studies use synthetic or saw bone models in comparing the biomechanical characteristics of these implants. ${ }^{3,5,8,9}$ In our study, load to failure values were indeed noted to be higher in both the Hybrid LCP group and the bone cementaugmented group as compared with the standard DCP group, similar to the results of previous studies.

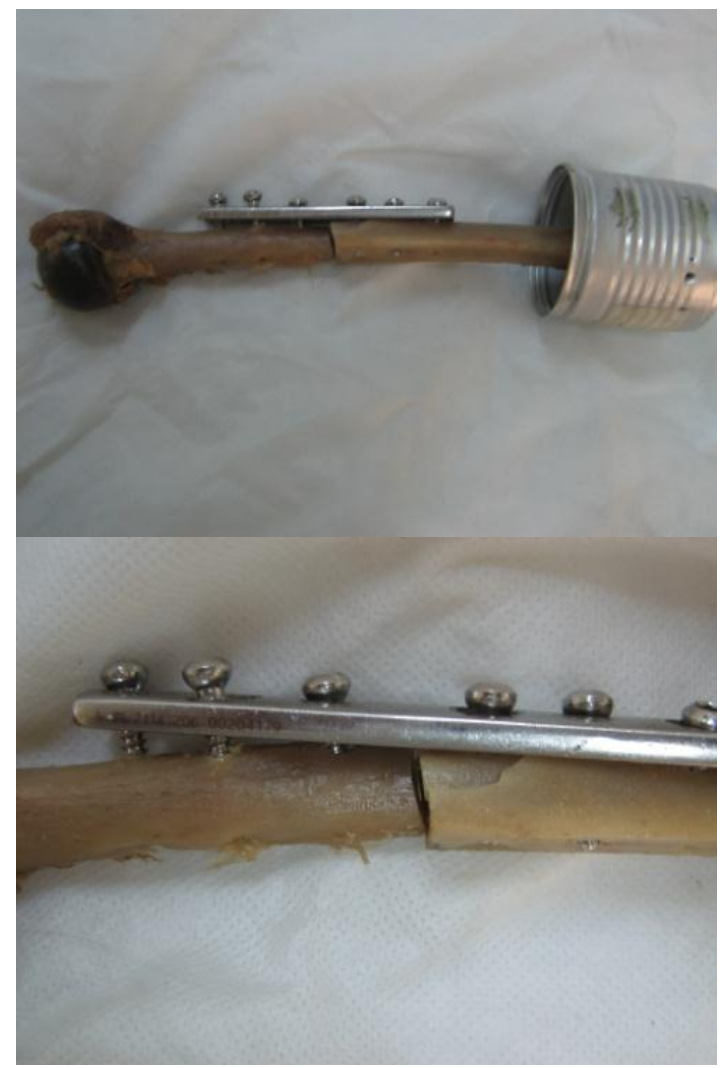

Figure 11. Pattern of screw pull-out in the regular DCP group tested in torsion

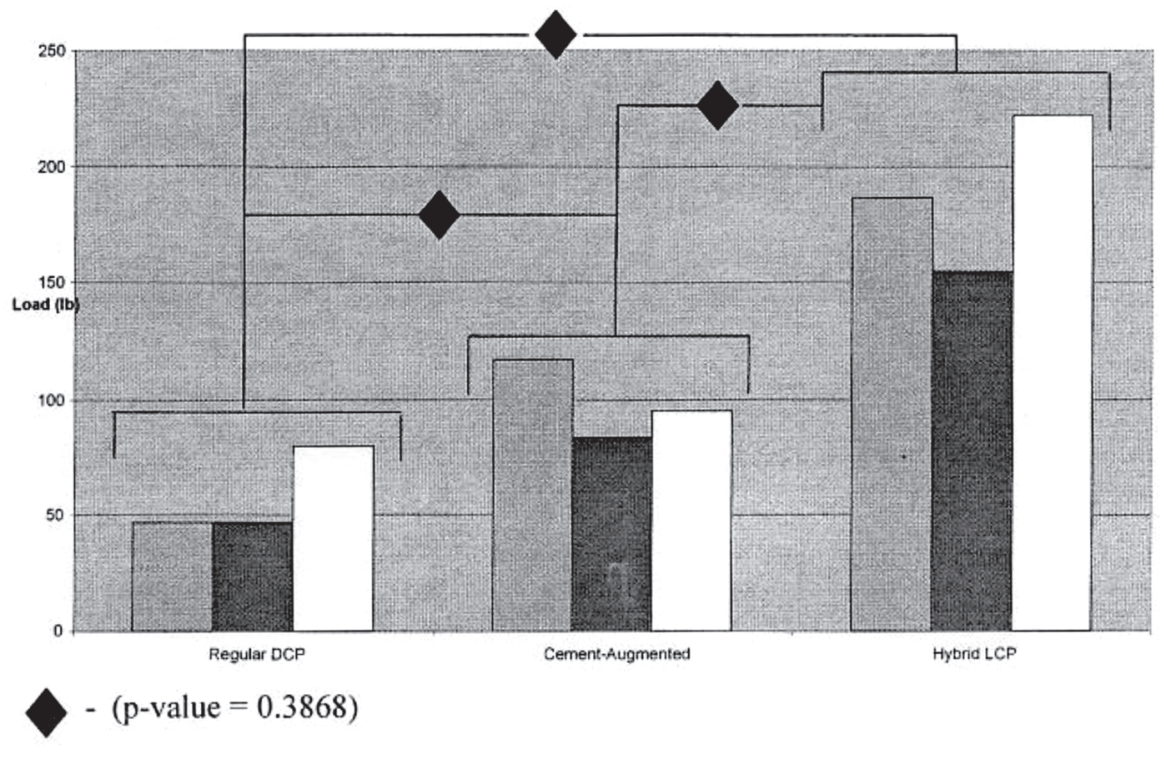

Figure 10. Mean load to failure values in four-point anterior-posterior bending by implant construct 
A. Hybrid LCP Group
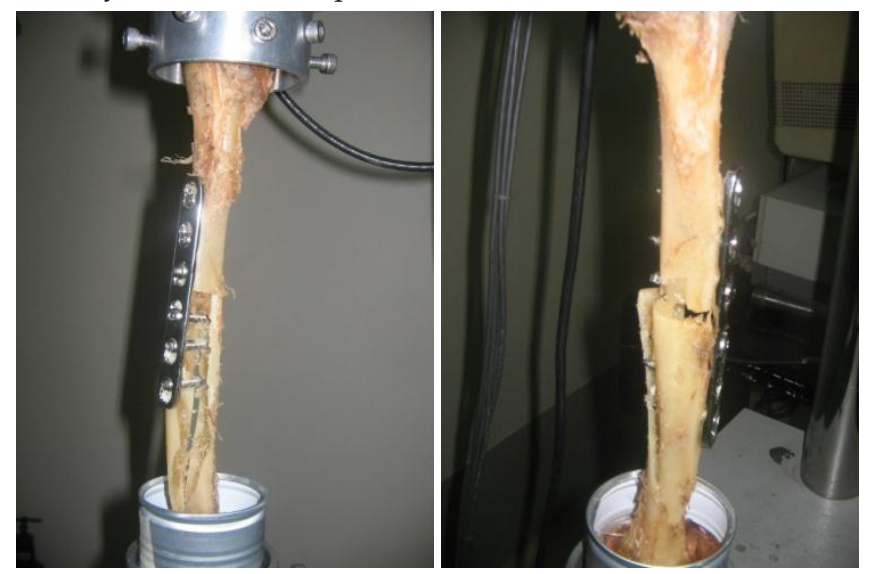

B. Bone cement-augmented DCP group

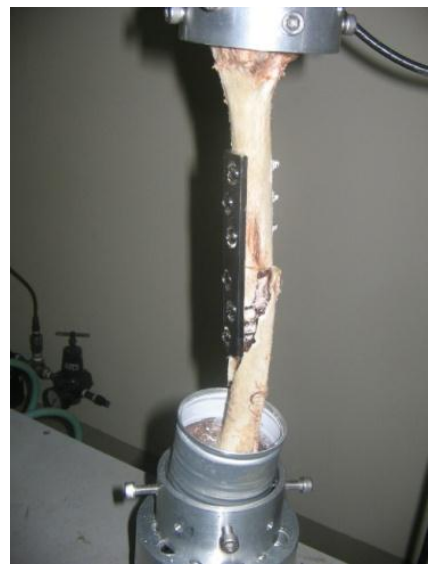

Figure 12. Pattern of bone failure in the bone cementaugmented DCP group and the Hybrid LCP group tested in torsion

Screw purchase in the bone is the major limitation of plate fixation in the osteoporotic humerus. Although PMMA has relatively poor adhesion to bone, it can intrude into its cancellous structure, resulting in a stronger composite after the cement polymerizes. Polymethylmethacrylate cement has been used to augment screw fixation, ${ }^{14}$ and if applied properly, makes a better construct in an osteoporotic humerus. Screw pull-out strengths of implants were found to be higher when augmented with bone cement as compared with conventional bone-construct. Although recent studies have supported the use of the LCP system in osteoporotic bone, reports of implant failure using this system have also been documented. ${ }^{15}$

Gardner et al. compared the LCP system to the standard DCP in anterior-posterior, medio-lateral, and torsional strain using cadaveric human radii. They described a subtle increase in antero-posterior and torsional strain in the locked compression plating group over dynamic compression plating in this group. ${ }^{16}$ Our biomechanical tests showed that the screw fixation with the hybrid locked plate resulted in a construct with superior stiffness compared with the standard DCP and cement-augmented constructs in torsion and four-point bending, as evidenced by their higher load to failure values. However, the biomechanical test failed to show the superiority of the Hybrid LCP constructs over the bone cement augmented constructs in terms of screw pullout strength. Screw pull-out did not occur in both the Hybrid LCP and bone cement-augmented DCP group. Bone fracture in both groups causing failure in the bone-implant construct interface was observed before any pull-out of the screws could be noted in both the Hybrid LCP and bone cement-augmented DCP group. Also, within the elastic range of the osteoporotic bone models in our study, screw purchase on the bone was acceptable for both the Hybrid LCP and bone cement-augmented DCP groups. Expected outcomes of screw pull-out, normally seen as the mode of failure in the clinical setting with the use of the standard plating technique, ${ }^{16}$ were only seen in the group of the standard DCP when loaded in torsion/external rotation. This was not observed in the Hybrid LCP and bone cementaugmented group. However, one unusual mode of bone fracture was observed in our study: a split of the humeral shaft into anterior and posterior fragments through the holes of the locking screws and the bone cement augmented DCP. This fracture pattern was similar to the two cases of complications using the LCP system in osteoporotic proximal humeral shaft fractures reported by Hall et al. ${ }^{16}$ In their study, they believed that the combination of a number of parallel, rigid locking screws in an osteoporotic humeral shaft can create a linear stress riser which can be a potential risk factor for catastrophic failure resulting in "fissuring" or splitting of the humeral shaft as a result of a less significant rotational stress. This may explain the fracture pattern seen in the majority of our specimens.

Although the use of the locking plate system in osteoporotic bones has its proven advantages, cement augmentation may still be an alternative if we desire to improve the screw purchase in osteoporotic bones. In fact, previous studies comparing PMMA construct and quadricortical construct using the fibula as strut graft within the intramedullary canal of the osteoporotic humerus have shown comparable screw purchase of the former compared to the latter. ${ }^{15}$ However, reports of deleterious effects on the blood supply of the bone and on healing with cement augmentation, especially if it extrudes into the fracture site, should also be considered.

One of the major theoretical advantages of the locking plate technology is the preclusion of compressing the plate to the bone, thereby avoiding subsequent disruption of the periosteal cortical blood supply. The use of the unlocked screws (Hybrid method) in the LCP system also has its advantages. It may allow compression across the fracture site, and if micromotion is eliminated and absolute rigidity is 
achieved, primary healing and direct fracture remodeling may occur. A combination of screw types in the LCP system may be preferable in situations in which osteoporosis is present or in which a compression screw is used as a reduction aid. ${ }^{3}$

Biomechanical investigation has shown the efficacy of using bone cement augmentation and the use of the Hybrid LCP system to improve screw fixation in osteoporotic bones of the humerus. These techniques are appropriate when the bone is osteopenic or when there is established non-union especially when previous fixation methods have failed. Hybrid locking screw constructs are substantially more expensive than the traditional unlocked screws, ${ }^{3}$ and it appears that at least bone cement-augmented constructs are biomechanically acceptable in the fixation of osteoporotic bones of the humerus. Additional biomechanical and comparative clinical studies are necessary to validate these concepts, but the use of either the bone cement-augmented constructs and the Hybrid LCP constructs may provide an additional clinical tool for the treatment of fractures in patients with poor bone quality.

\section{Conclusion}

Our biomechanical test showed that in the cadaveric osteoporotic humeral shaft fracture model, fixation with the Hybrid LCP system provided a stiffer construct compared with the standard DCP and bone cement-augmented DCP in torsion/external rotation in terms of load to failure which was statistically significant. Load to failure values were higher for the bone cement-augmented DCP compared with the regular DCP, but were not statistically significant.

Load to failure values, however, were not statistically significant among the three groups of construct in the anterior-posterior four-point bending tests.

Bone failure, but not screw pull-out, was observed in the Hybrid LCP system and the bone cement-augmented DCP group when loaded in torsion. Both the LCP system and the bone cement-augmented constructs may provide greater screw purchase to the osteoporotic humerus in our study. Other modes of failure, particularly bone fracture, were observed which warrant further investigation.

\section{Limitations of the Study}

Since this is a biomechanical study, results obtained from the cadaveric fracture models may have limited application in the actual clinical setting. The importance of soft tissue in fracture healing must also be considered in the clinical setting. ${ }^{8}$ Cadaveric bone specimens were used in this study based on the previously described protocol by Wright. ${ }^{15}$ Although we did all the necessary procedures to standardize the specimens, the variability in cadaveric bones still had an effect on our biomechanical testing, as opposed to using sawbones which are more standardized. Due to the limited supply of cadaveric bones, we were only able to utilize three bones per treatment group $(n=3)$. A larger sample size ( $n=4$ as computed in the statistics) would have been more ideal in order to minimize variability in the results and increase the power of the study. Cyclic loading was not performed in this study. Only load to failure values were measured due to the limitation of the Universal Testing Machine which could not perform cyclic loading at the time the study was conducted. Comparison of screw pull-out strengths was not tested among the constructs because bone failure occurred first in the majority of the osteoporotic specimens. Screw pull-out may be further investigated using a synthetic bone test medium such as homogenous polyurethane foam rather than an osteoporotic bone model.

\section{References}

1. Cornell CN. Internal fracture fixation in patients with osteoporosis. J Am Acad Orthop Surg. 2003; 11(2):109-19.

2. Egol KA, Kubiak EN, Fulkerson E, Kummer FJ, Koval KJ. Biomechanics of locked plates and screw. J Orthop Trauma. 2004; 18(8):488-93.

3. Gardner MJ, Griffith MH, Demetrakopoulos D, et al. Hybrid locked plating of osteoporotic fractures of the humerus. J Bone Joint Surg Am. 2006; 88(9):1962-7.

4. Hak DJ, Hazelwood SJ, Cordero G. Comparison of the AO locking plate with the standard limited-contact dynamic compression plate (LC-DCP) for fixation of osteoporotic humeral shaft fractures. Orthopedic Trauma Association Scientific Poster. California, USA. 2003.

5. Levy JC, Latta LL, Kalandiak SP. Locked vs. unlocked plating for humeral shaft nonunions: a biomechanical study. Am Acad Orthop Surg Scientific Poster. Chicago, USA. 2006.

6. Ring DC, Kloen P, Kadzielski JJ, et al. Locking compression plates used for delayed unions and nonunions of the diaphyseal humerus. Orthopedic Trauma Association Scientific Poster. New York, USA. 2002.

7. Gardner M, Brophy R, Campbell DA, et al. The biomechanical behavior of locking compression plates in osteoporotic bone. Am Acad Orthop Surg Scientific Poster. Washington, USA. 2005.

8. Larsson S, Bauer TW. Use of injectable calcium phosphate cement for fracture fixation: a review. Clin Orthop Relat Res. 2002; 395:23-32.

9. Ellis $T$, Bourgeault CA, Kyle RF. Screw position affects dynamic compression plate strain in an in vitro fracture model. J Orthop Trauma. 2001; 15(5):333-7.

10. ElMaraghy AW, ElMaraghy MW, Nousiainen M, Richards RR, Schemitsch EH. Influence of the number of cortices on the stiffness of plate fixation of diaphyseal fractures. J Orthop Trauma. 2001; 15(3):18691.

11. Rubel IF, Kloen P, Campbell D, et al. Open reduction and internal fixation of humeral nonunions: a biomechanical and clinical study. J Bone Joint Surg Am. 2002; 84-A(8):1315-22.

12. Jazrawi L, Bai B, Simon JA, Kummer FJ, Birdzell LT, Koval KJ. A biomechanical comparison of Schuhli nuts or cement augmented screws for plating of humeral fractures. Clin Orthop Relat Res. 2000; 377:235-40.

13. Tornkvist H, Hearn TC, Schatzker J. The strength of plate fixation in relation to the number and spacing of bone screws. J Orthop Trauma. 1996; 10(3):204-8.

14. Cameron HU, Jacob R, Macnab I, Pilliar RM. Use of polymethylmethacrylate to enhance screw fixation in bone. J Bone Joint Surg Am. 1975; 57(5):655-6.

15. Wright TW, Miller GJ, Vander Griend RA, Wheeler D, Dell PC. Reconstruction of the humerus with an intramedullary fibular graft: a clinical and biomechanical study. J Bone Joint Surg Br. 1993; 75(5):804-7.

16. Hall JA, Phieffer LS, Mckee MD. Humeral shaft split fracture around proximal humeral locking plates: a report of two cases. J Orthop Trauma. 2006; 20(10):710-4. 